\title{
Ética, capital intelectual y gestión del conocimiento
}

José Manuel Saíz Álvarez*

Ethics, Intellectual

Capital \& Knowledge

Management

Éthique, Lapital

Intellectue/ et

Gestion de la

Connaissance

Etica, capital intelectual

e gestãu do conhecimento
*Doctor “cum laude" en Ciencias Empresariales, Universidad Autónoma de Madrid (España) y Doctor "cum laude" en Sociología, Universidad Pontificia de Salamanca (España); profesor de la Universidad Antonio de Nebrija y de la Universidad Pontificia de Salamanca (Campus de Madrid) 


\section{RESUMEN}

Resultado del proceso de globalización económica actual, unido al inicio de la denominada «Primera Crisis Global (PCG), cada vez es mayor la importancia del capital intelectual en las organizaciones, tanto por las ventajas competitivas que genera para la organización, como por permitir que la empresa pueda lograr posiciones de liderazgo sostenible en el tiempo. Dicho capital intelectual se inserta dentro de las organizaciones siguiendo una doble perspectiva basada en la ética y en la gestión del conocimiento. El objetivo de este trabajo es analizar cuáles son las bases que, desde una gestión del conocimiento basado en la ética aplicada a la empresa, permiten construir organizaciones líderes en el mercado global.

\section{ABSTRACT}

As a result of the current economic globalization process, closely related with the onset of the First Global Crisis, organizational intellectual capital is increasing its importance as it brings better competitive advantages and it allows to reach sustainable leadership positions through years. This already stated intellectual capital flows within organizations following a double- side perspective based on ethics and knowledge management. This paper aims at determining which the basis are, from a knowledge management ethical perspective applied to organizations, to shape leading organizations for a global market.

\section{RESUMÉÉ}

Les résultats du processus actuel de mondialisation économique qui coïncident avec le début de la dénommée "première crise globale" (PCG), révèle l'importance de plus en plus grande que rencontre le capital intellectuel dans les organisations. Son importance se développe tant pour les avantages concurrentiels générés pour les entreprises que pour permettre que celles-ci se retrouvent dans des positions durables de leadership. Le capital intellectuel est inséré au sein des organisations à la suite d'une double perspective basée sur l'éthique et la gestion des connaissances. L'objectif de ce travail consiste à analyser la manière dont les bases d'une gestion de la connaissance fondée sur l'éthique appliquée à l'entreprise permettent de construire des organisations de premier plan international.

\section{RESUMO}

Resultado do processo de globalização econômica atual, ligado ao inicio da chamada «Primeira Crise Global (PCG), cada vez é maior a importância do capital intelectual nas organizações, tanto pelas vantagens competitivas que gera para a organização, quanto por permitir que a firma possa atingir posições de liderança sustentável através do tempo. Esse capital intelectual se insere dentro das organizações seguindo uma dupla perspectiva baseada na ética e na gestão do conhecimento. O objetivo deste trabalho é analisar quais são os fundamentos que, desde uma gestão do conhecimento baseada na ética aplicada à empresa, permitam construir organizações líderes no mercado global.

\section{Palabras claves}

\author{
Capital intelectual \\ Gestión del conocimiento \\ Etica
}

\section{Key words}

Intelectual capital

Knowledge management Ethics

\section{Capital intellectuel \\ Gestion de la \\ Connaissance Ethique}

\section{Palavras-chave}

\author{
Capital intelectual \\ Gestão do conhecimento \\ Ética
}




\section{INTRODUCción}

L a nueva economía actual, en un doble sentido tanto McLuhaniano con su aldea global, creada a partir de la interconexión mundial gracias a las Tecnologías de la Información y la Comunicación, TIC, (McLuhan, 1989), como Druckeriano con sus predicciones sobre la llegada de una economía basada en información y conocimiento más que en la mera producción de bienes y servicios; lleva a que se pueda hablar de una era de trabajo basada en el conocimiento (Knowledge-based worker age) (Drucker, 1969) En dicha economía el conocimiento se generaliza rápidamente gracias al uso de las TIC, lo que provoca, junto con la movilidad del capital intelectual, que se mueve y colabora entre distintas organizaciones, una dispersión del conocimiento
(Knowledge Spillover) que beneficia tanto al individuo como a la empresa y a la sociedad en su conjunto.

El objetivo de este trabajo es analizar cuáles son las bases que, desde una gestión del conocimiento, basado en la ética aplicada a la empresa, permiten construir organizaciones líderes en el mercado global. La generalización de las TIC, junto con el rápido crecimiento de las denominadas economías emergentes, formadas por la combinación de los llamados BRIC (Brasil, India, China y Rusia) los EAGLE latinoamericanos (Brasil y México) y Nidos de EAGLE (Colombia, Chile y Perú), han Ilevado hacia un crecimiento tanto en la competencia entre instituciones como en el intercambio de capital intelectual entre las instituciones. 


\section{EL CONOCIMIENTO COMO UN ACTIVO INTANGIBLE ILIMITADO}

egún Romer (1995, citado por Davenport y Prusak, 2001) el conocimiento es el único activo que es ilimitado al ir creciendo con su uso. Incremento que va generando valor no solamente para el individuo (valor endógeno) sino también para la organización (valor exógeno). Valores que afectan positivamente tanto a la actividad y los resultados empresariales, como a la formación de un fondo de comercio que favorece principalmente a los accionistas de la misma. Valores unidos a un conocimiento, que se puede clasificar en tácito, cuando es un conocimiento no verbalizable, intuitivo y no articulado (Polanyi, 1958), y explícito, cuando es transmisible de manera formal, con un lenguaje sistemático y puede incluir hechos explícitos, proposiciones axiomáticas y símbolos (Kogut y Zander, 1992). Por ello, tanto en la persona como en las organizaciones se dan ambos tipos de conocimiento de manera simultánea.

Por otro lado, para Moran y Ghoshal (1996) todos los recursos, incluido el conocimiento, se crean a partir de dos procesos genéricos: mediante la combinación de recursos y, sobre todo, mediante un proceso interactivo de intercambio de los mismos, incluyendo el capital intelectual, cuando difiere el grado de conocimiento entre los agentes económicos que interaccionan entre sí (Jonavovic y Rob, 1989). Conocimiento que es creado a través de cambio incremental (Nahapiet y Ghoshal, 1998). Por ese motivo, las organizaciones que se encuentran en interacción entre ellas, o que pertenecen a asociaciones empresariales, tienen una mayor probabilidad de generar un conocimiento que favorece tanto a la producción como a la comercialización de los productos y servicios creados en ellas. Resultado de todo ello, las organizaciones endogámicas suelen convertirse a medio plazo en empresas estancadas que, en casos extremos, se convierten en organizaciones de supervivencia, que incluso llegan a desaparecer.

El conocimiento nacido dentro de las organizaciones se difunde hacia la sociedad, lo que genera efectos beneficiosos para toda ella. Unos efectos

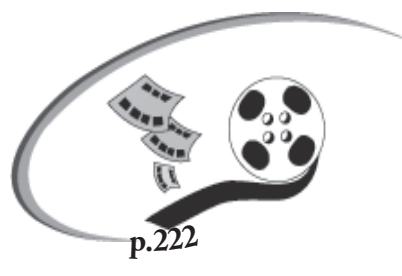
que provoca situaciones óptimo paretianas, o en su caso, subóptimas si la organización no alcanza una posición de liderazgo en su sector. Conocimiento social que, a su vez, enriquece al conocimiento nacido de las empresas, al darse en un sentido dinámico.

Por su parte, además del conocimiento individual que beneficia a las organizaciones, se distingue también un conocimiento social, el cual, según Nahapiet y Ghoshal (1998), puede ser de dos tipos al igual que el individual: tácito o explícito. El conocimiento social tácito, denominado también conocimiento colectivo, incluye las costumbres y la cultura de una comunidad local, desde el núcleo familiar (incluyendo familias monoparentales) hasta empresas y comunidades. Por su parte, el conocimiento social explícito, denominado conocimiento objetivado, constituye "la forma más avanzada del conocimiento" (Boisot, 1995, citado por Nahapiet y Ghoshal, 1998, p. 247), al estar dentro de equipos de trabajo especializados, crea ventajas competitivas de primer orden o de rango 
superior mediante la interacción de sus miembros. Nacido tras el trabajo seminal de Polanyi (1958) y generalizado por la Escuela Austriaca de Economía ${ }^{2}$ y los trabajos de la Escuela Institucionalista ${ }^{3}$, Economía Industrial y Geografía Económica, el concepto de conocimiento tácito es criticado por Perraton y Tarrant (2007). En un estudio realizado para 89 empresas de alta tecnología situadas en la provincia china de Jiangsu, Wang y Wang (2012) demuestran que la combinación de conocimiento tácito con explícito facilita tanto la innovación (Koenig, Lorenz y Zilibotti, 2012) como el desempeño dentro de la organización, debido a que compartir conocimiento explícito acelera los procesos de innovación y mejora los resultados financieros, mientras que compartir el conocimiento tácito influye sobre todo en la calidad de la innovación y el desempeño operacional.

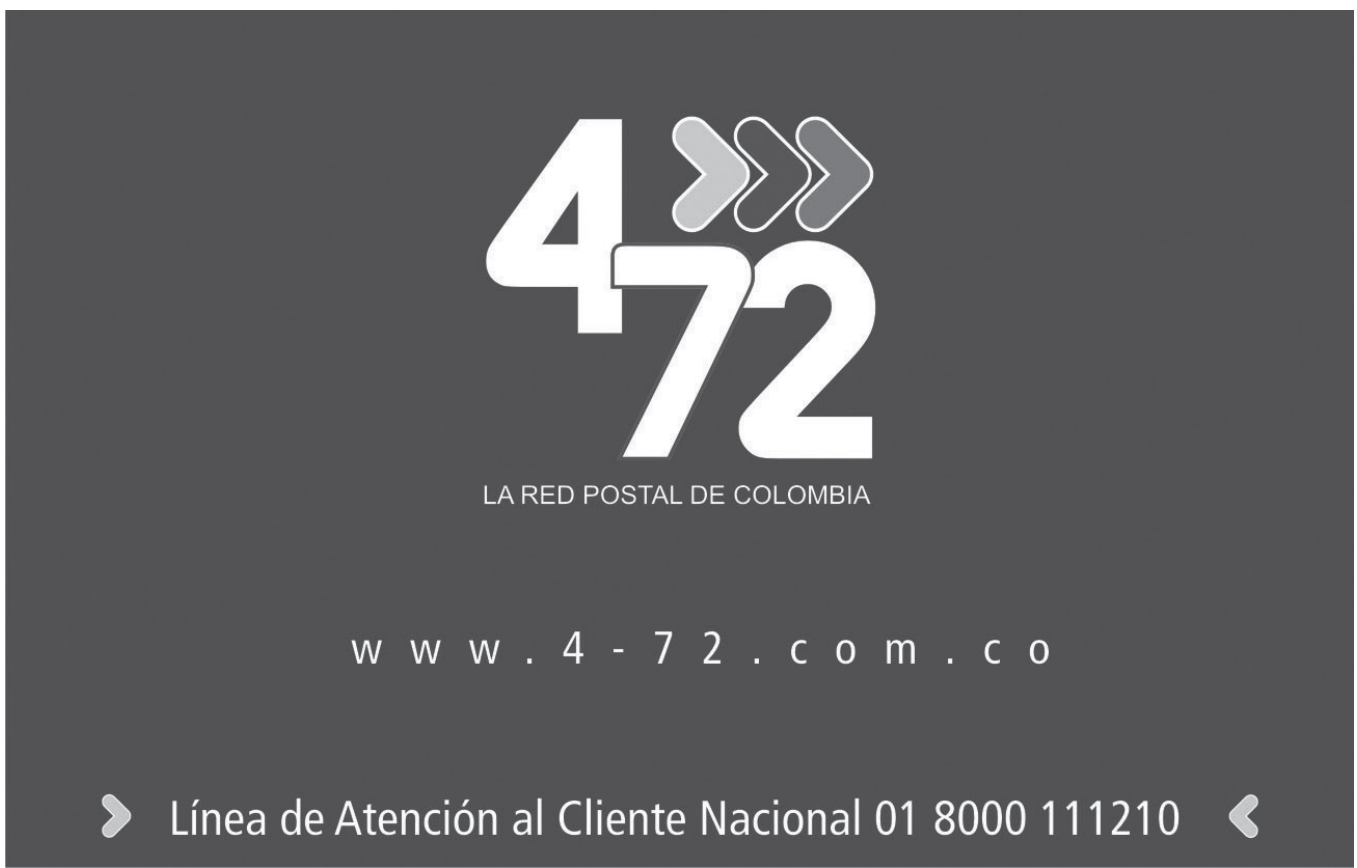

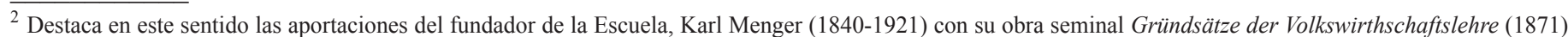
(Principios de Economía Política), obra que fue continuada por Eugen von Böhm-Bawerk (1851-1914). Además de estos autores como máximos exponentes de esta escuela destacan: Friedrich von Wieser (1851-1926), Ludwig von Mises (1881-1973), Friedrich A. Von Hayek (1899-1992), Fritz Machlup (1902-1983) e Israel Kirzner (1930-)

${ }^{3}$ Esta Escuela se basa en dos grandes principios: (1) Crítica a la economía neoclásica y (2) El enfoque histórico-cultural de la economía, ya que "la sociedad contemporánea es un complejo de instituciones o formas habituales para organizar y regular la conducta de los individuos. En cierto modo, el desarrollo de las instituciones es un proceso competitivo, y su resultado determina qué formas de conducta son aceptadas y aprobadas por la comunidad" (Urbano, Díaz y Hernández, 2007, p.192). Como máximos representantes de esta Escuela de Pensamiento destacan Thorstein Veblen, a partir del libro seminal Teoría de la clase ociosa (1899), John R. Commons, Gunnar Myrdal, Clarence Ayres (1891-1972), Adolf Berle (1895-1971) y John Kenneth Galbraith (1908-2006). Dentro de la Nueva Economía Institucional destacan Douglas North y Oliver Williamson
} 


\section{EL CÍRCULO VIRTUOSO DE LA GESTIÓN DEL CONOCIMIENTO}

L a gestión tiene como objetivo asegurar la racionalidad del proceso de toma de decisiones empresariales para lograr un objetivo común para lo cual ha de planificar, organizar, liderar y controlar todo el proceso productivo (Mitja, 2011). De esta manera se logran de forma simultánea tres objetivos: un objetivo técnico mediante la unión de varios subprocesos dentro de un proceso productivo, cuya complejidad técnica varía en función del producto o servicio a prestar al cliente final; un objetivo empresarial caracterizado por el uso de buenas prácticas y comportamiento ético para lograr los objetivos establecidos, y un objetivo social al tener toda actividad económica un impacto social, ya sea endógeno en los propios profesionales y trabajadores que ejercen su actividad profesional en la organización como en las externalidades generadas, tanto en el entorno personal y familiar de los Stakeholders, como en la sociedad en su conjunto. En este sentido, cuanto mayor ex ante sea el tamaño de la empresa, creciente será ex post el impacto social de dicha actividad empresarial.

Por su parte, el conocimiento se puede definir como el conjunto de "aquellos factores que tienen el poten-

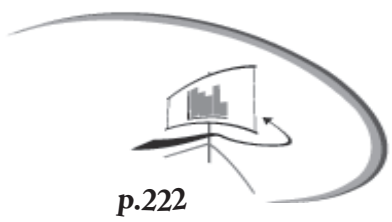
cial de influir tanto al pensamiento como al comportamiento humano, lo que determina la producción y el control del sistema productivo"

(Mitja, 2011:)

e incluye, entre otros, el diseño de la organización, las habilidades gestoras y productivas de los profesionales y trabajadores, la imagen corporativa y de marca, incluyendo incluso la marca persona y los tipos de conocimiento (tácito o descodificado y explícito o codificado)(Hall y Andriani, 2003: p. 145) (Nonaka y Takeuchi, 1995).

Se considera que el conocimiento es una forma de inteligencia abstracta que tiene un triple impacto sobre el individuo, sobre la organización en la que trabaja y sobre la sociedad, al unirse la capacidad para aprender (inteligencia) y la oportunidad para hacerlo.

El conocimiento presenta así seis características, al ser:

- Acumulativo, ya que al poderse sumar nuevas experiencias y estudios sobre un tema particular o un área de conocimiento más general, el conocimiento se va haciendo más grande tanto para el individuo como para la empresa. Como resultado, la suma de experiencia profesional y formación académica constituyen un valor para el individuo. de lo que se beneficia tanto la empresa como la sociedad en su conjunto.

- De rápida obsolescencia, por lo que el conocimiento ha de estar continuamente adaptado a nuevas teorías y experiencias. La velocidad en dicha obsolescencia varía en función del sector que consideremos, siendo más rápida en aquellos sectores más abiertos al exterior, al tener que competir a escala global debido a la globalización económica.

- Espontáneo, sobre todo al inicio de nuevos procesos productivos que han nacido como 
resultado de problemas, disfunciones e ineficiencias que había que resolver.

- Integrador, ya que actúa en relación con otros tipos de conocimiento relacionados entre sí. A mayor integración, mayor facilidad para adaptarse al cambio.

- Subjetivo, debidoaquevaríalainterpretacióndel conocimiento en función de las circunstancias y el ambiente social, económico, empresarial y político del agente económico que la utiliza.

- Transmisible, lo que permite una rápida difusión del mismo, sobre todo a partir del uso de las Tecnologías de la Información y la Comunicación (TIC).

Figura 1. El ciclo virtuoso de la gestión del conocimiento

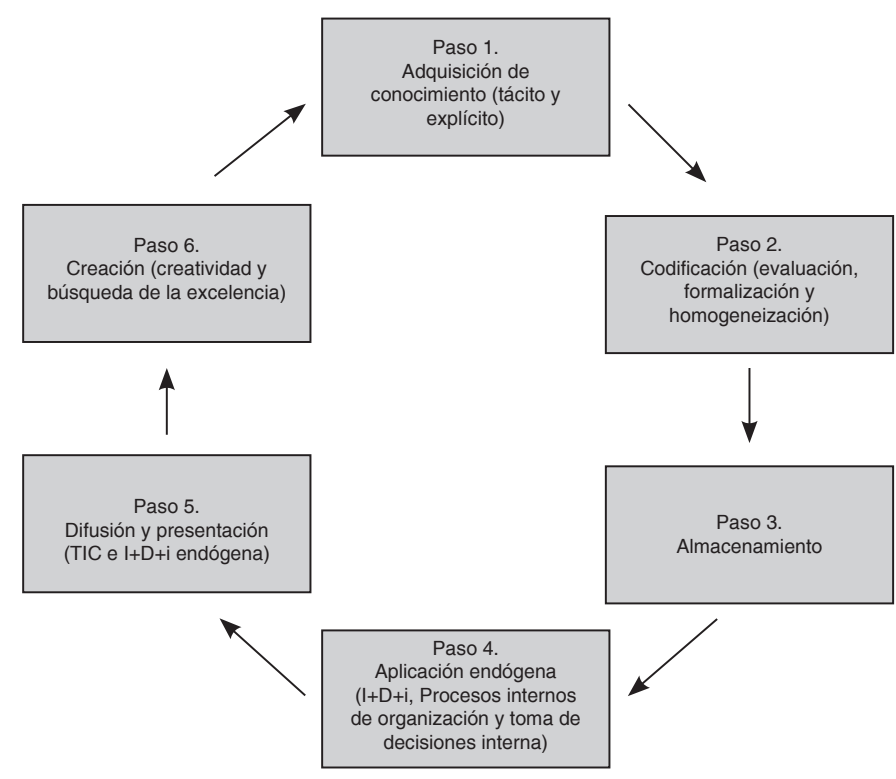

Fuente. Elaboración propia.
Se puede concebir a la gestión del conocimiento como el conjunto de prácticas y estrategias empresariales, basadas en conocimiento tácito y explícito (Nonaka y Takeuchi, 1995) y en la coordinación de personas, tecnologías y procesos dentro de una organización (Mishra, 2009), que tienen como objetivo maximizar al conocimiento como recurso productivo de la empresa (Becerra-Fernández y Sabherwal, 2010). Recurso productivo que permite a la organización alcanzar posiciones de liderazgo en su sector (o sectores) en los que desarrolla su actividad empresarial, así como atraer al mejor capital intelectual de diferentes organizaciones para así poder competir con éxito en los mercados internacionales. Podríamos hablar así de un ciclo virtuoso de la gestión del conocimiento que consta de seis etapas (figura 1) si se desea que éste sea sostenible en el tiempo.

Una de las claves que explica el por qué las organizaciones tienen éxito viene dado por la existencia de un proceso creativo autoalimentado por los recursos productivos de la organización en una constante búsqueda de la excelencia mediante el uso de las TIC y la aplicación de I+D+i endógena, esto es, generada dentro de la organización o en colaboración en grupos internacionales formados por empresas, universidades y centros de investigación. Un círculo virtuoso cuya ruptura será cada vez más difícil en el tiempo a medida que los integrantes del mismo vayan obteniendo buenos resultados de dicha alianza estratégica. 


\section{ÉTICA Y CAPITAL INTELECTUAL}

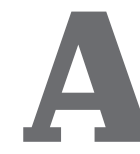
partir del modelo de gestión del conocimiento ideado por DeLone y McLean (1992), Leung et al (2012), amplían el mismo para diferentes niveles jerárquicos en la organización, llegando a la conclusión que dicha gestión

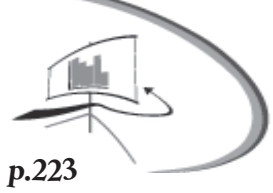

ha de ser dinámica y está en un continuo proceso de adaptación. Por ello, en un mundo globalizado es importante saber compartir conocimiento de una forma coordinada (Bradfield, Gao y Soltan, 2007), para así maximizar su impacto tanto económico y empresarial, como social.

Por su parte, Hsu y Sabherwal (2012) analizan la relación entre la gestión del conocimiento y el capital intelectual, teniendo en cuenta su impacto en tres aspectos: las capacidades dinámicas tanto de la organización como del capital intelectual que trabaja en ella, el nivel de eficiencia conseguido en la organización y la capacidad de innovación por parte de los stakeholders de la empresa. Como resultado de todo ello, tanto la formación como la experiencia práctica del capital intelectual que trabaja en la organización influyen en las políticas de gestión del conocimiento que está implementado o pretende incorporar la organización en un futuro; se generan capacidades dinámicas en la organización que la hacen más competitiva; se facilitan tanto la innovación como la gestión del capital en la empresa, y se desarrolla la innovación y la eficiencia en la empresa por parte del capital intelectual que desarrolla su actividad profesional en la misma.
Para lograr estos resultados es fundamental sentar bien las bases desde un principio. Inicios que para muchas organizaciones empresariales, sobre todo familiares, son modestas. Como resultado y como el conocimiento interiorizado por parte del capital intelectual que trabaja en la organización mejora la toma de decisiones, se produce un continuo impulso tanto para interiorizar conocimiento como para optimizar la buena gestión del mismo.

Para Davenport y Prusak (2000), disponer de conocimiento es más importante que la mera inversión en I+D+i. Los procesos de generación de nuevas tecnologías se caracterizan por tener, por lo general, este proceso, basado en el método de prueba-error, un período de maduración elevado (salvo en los sectores muy dinámicos y competitivos) para así poder recuperar la inversión, y por la necesidad de disponer de un capital intelectual altamente especializado y profesionalizado.

Al estar basada la nueva economía en el conocimiento y en la aplicación práctica del l+D+i generado internamente o adquirido por la organización, son fundamentales los aspectos éticos tanto en su generación como en su implantación. Entre las políticas de ética empresarial que puede aplicar la organización, destacan la siguiente trilogía:

- Ética transaccional, que se caracteriza por la interacción dinámica entre distintos agentes económicos situados en distintas áreas productivas o comerciales de la empresa, o fuera de la misma. En la ética transaccional 
se engloban las políticas de Responsabilidad Social Corporativa (RSC) o Empresarial (RSE) y la responsabilidad fiduciaria.

- Ética participativa, que se define por la democratización en la toma de decisiones, así como en la implantación de políticas éticas en la empresa. Dentro de este apartado destacan las políticas de buen gobierno o gobernanza económica realizadas por la organización.

- Ética de reconocimiento, que se concreta en la imagen proyectada por la empresa hacia el exterior, tal y como es reconocida por los Stakeholders. Dentro de este tipo de ética destaca la política de transparencia, la marca y la reputación corporativa.

El aumento de las capacidades de gestión del conocimiento (tanto en la mejora del conocimiento, como en la optimización en el uso del conocimiento) facilita la creación de I+D+i y mejora el capital intelectual que trabaja en la organización. Como resultado, las empresas mejoran resultados y logran mantener su posición de liderazgo de forma sostenible en el tiempo.

Desde una perspectiva práctica, la puesta en marcha de estrategias empresariales basadas en la ética viene dada a partir de:

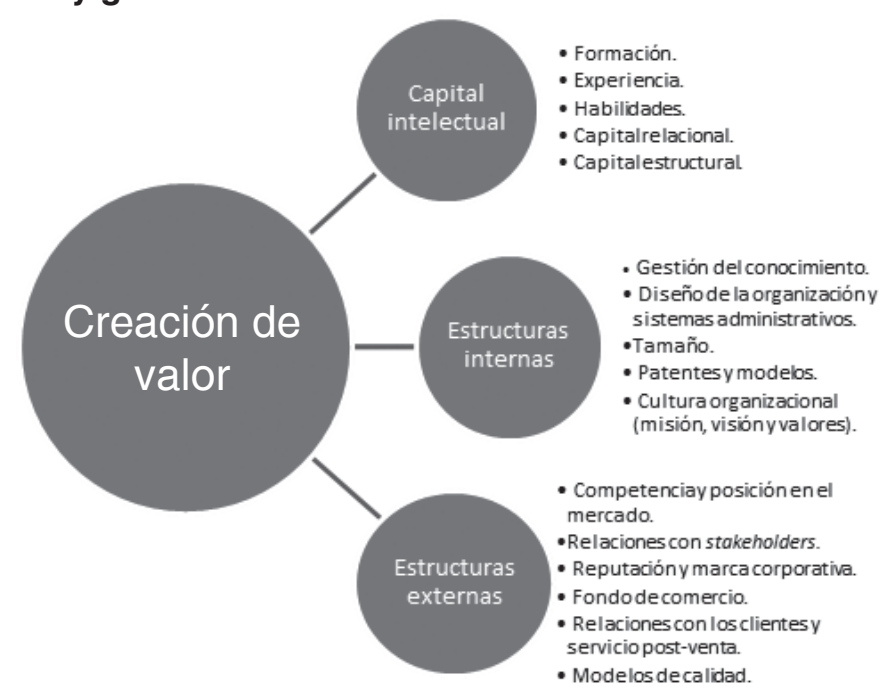

Fuente. Elaboración propia a partir de Hussi (2003).

\section{Figura 2. Creación de valor y gestión del conocimiento}

- El inicio de políticas formativas dentro y fuera de la organización que sean acordes con la misión, la visión y los valores de la empresa.

- La difusión dentro de la organización (difusión endógena) de las políticas y estrategias corporativas que va a utilizar la empresa en aras de su mayor consolidación en el mercado.

- El incentivo de estrategias tanto de fusiones empresariales (pliopolios negativos) (Saiz y Olalla, 2010), como de adquisiciones de empresas, sea mediante compra directa de las participaciones sociales en caso de cooperativas y sociedades limitadas, o sea mediante Ofertas Públicas de Adquisición (OPA), hostiles o no, si la organización cotiza en mercados financieros.

- La puesta en marcha de equipos pluridisciplinares altamente integrados y con un objetivo común, plenamente integrados con la misión, la visión y los valores de las empresas, lo que permite interiorizar la cultura corporativa de la empresa y difundirla hacia el exterior, principalmente hacia clientes y socios.

- El inicio de políticas de Responsabilidad Social Corporativa (RSC) o Empresarial (RSE) porque influyen positivamente tanto en la imagen y marca corporativa, como en la reputación empresarial. 


\section{ANÁLISIS DAFO DE LA ÉTICA EMPRESARIAL}

$\mathbf{E}$ $\mathrm{n}$ un mundo altamente competitivo como el actual, y más en aquellas naciones más afectadas por la Primera Crisis Global (PCG), la puesta en marcha de políticas de ética empresarial

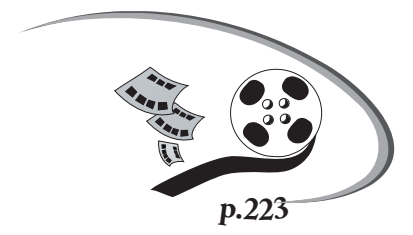

van a tener un impacto distinto en función de la características de las organizaciones y de su posición competitiva en el sector. Por ello, no habrá una única receta de aplicación, sino que ésta tendrá que ir adaptándose a cada situación en la que se encuentre la empresa. Para tener una visión global del impacto de estas políticas empresariales vamos a realizar un análisis DAFO (Debilidades, Amenazas, Fortalezas y Oportunidades) de las mismas.

\subsection{Debilidades}

Entre las debilidades más relevantes a la hora de implantar políticas de ética empresarial, hay que destacar las siguientes:

- La necesidad de un mínimo tamaño crítico de la organización, una vez que ha superado los primeros estadios de desarrollo marcados por la supervivencia.

- Toda política de ética empresarial necesita fondos para desarrollarse. Aunque lo ideal es que sean fondos propios de la organización, con la realización de ampliaciones blancas del capital social de la empresa (ampliaciones con cargo a reservas, ya sea legal, estatutaria o voluntaria), también es factible la aplicación de préstamos ad hoc para actividades solidarias. Préstamos que, a su vez, aumentan el riesgo lo que constituye una debilidad para la organización.

- Para poder aplicar políticas de ética empresarial el capital intelectual que trabaja en la organización ha de tener interiorizada tanto la misión, como la visión y los valores de la misma. Sin esta interiorización no se dará el efecto difusión, tanto hacia el interior como hacia el exterior, que es necesario para maximizar el impacto de la política ética.

- Existe la tendencia a que únicamente las grandes corporaciones son las que se ven más incentivadas para realizar políticas de ética empresarial, no así las Pequeñas y Medianas Empresas (PyMEs) ni las microempresas. Como resultado, el impacto de dichas políticas es limitado, al estar basado el tejido productivo y comercial de los países en PyMEs y microempresas, muchas de ellas familiares.

\subsection{Amenazas}

Las amenazas más sobresalientes para implantar políticas de ética empresarial tenemos las siguientes:

- Es especialmente difícil lograr unos elevados niveles de transparencia en entornos corruptos o altamente politizados. Como resultado, se hace necesario separar los intereses políticos de los económicos dentro de las organizaciones, para así garantizar un nivel de transparencia en la adopción de medidas que beneficia a los Stakeholders, en especial los clientes, y lograr así la mejora de la imagen corporativa. 
- Al buscar sus propios intereses, la existencia de intereses tanto de las oligarquías como de accionistas institucionales pueden desviar los objetivos de la organización.

- El éxito conseguido con las políticas de éti-ca empresarial puede llevar a que sean imitadas por el resto de competidores, lo que elimina la ventaja competitiva obtenida por la organización que inició dicho proceso.

- Si no se produce de forma simultánea una política de transparencia y de lucha contra la corrupción, los beneficios conseguidos con políticas de ética empresarial se verán contrarrestados. De hecho, conseguir una buena imagen, es una política de esfuerzo diario, mientras que se puede muy rápidamente dicho prestigio.

\subsection{Fortalezas}

Como fortalezas tras la correcta aplicación de políticas de ética empresarial se destacan las siguientes:

- Las organizaciones éticas son las que mejor compiten de forma sostenible en el mercado al verse reconocidas por los clientes, tanto actuales como futuros.

- La ética corporativa sienta las bases para una mejora de la imagen y marca corporativas, lo que atrae a nuevos clientes y al mejor capital intelectual existente en el mercado.

- La reputación corporativa y las políticas de buen gobierno favorecen la creación de valor dentro de la empresa, lo que favorece principalmente a los accionistas y da garantía de estabilidad a medio y largo plazo principalmente a socios y proveedores.

- El éxito conseguido con la aplicación de ética empresarial facilita el crecimiento a la empresa y permite, a medio plazo, iniciar con la puesta en marcha de grupos empresariales.
- La puesta en marcha de políticas de ética empresarial mejora el ambiente laboral en la empresa, lo que redunda en un incremento de la productividad marginal del trabajo. Esto beneficia especialmente a la corporación.

\subsection{Oportunidades}

Por último, como oportunidades para el diseño y la puesta en marcha de políticas de ética empresarial se destacan las siguientes:

- El éxito en el diseño y la puesta en marcha de políticas empresariales basadas en la ética lleva hacia un aumento en la calidad y el incremento de la cantidad y calidad de clientes y socios.

- Lamejora delEBITDA(Earnings before Interest, Taxes, Depreciation and Amortization), lo que fortalece a la empresa en el sector, permite a la organización alcanzar posiciones de liderazgo, es un instrumento para aumentar las reservas y la capitalización bursátil, siempre que cotice en bolsa de valores. Todo ello lleva hacia una mayor sostenibilidad corporativa a medio y largo plazo.

- La atracción del mejor capital intelectual existente en el mercado, lo que crea un efecto retroalimentado a medida que dicho capital va cumpliendo con los objetivos establecidos por la organización.

- Se fortalece la relación entre los profesionales con mayor experiencia laboral (On-the- job learning) (Ben-Porath, 1967; Heckman, 1976; Rosen, 1976) y aquellos que la tienen limitada o nula. De esta manera se produce en las organizaciones un efecto continuo de enseñanza-aprendizaje dentro del capital intelectual que trabaja en ella (Lucas, 2009). Un proceso dinámico que se ve favorecido cuando se han aplicado políticas de ética empresarial. 


\section{CONCLUSIONES}

P or su propia naturaleza, el conocimiento tácito y explícito está orientado hacia la acción y se va ampliando como activo en el tiempo. La introducción de políticas de ética empresarial en la organización favorece la generación de valor, así como la atracción de clientes, socios y capital intelectual. Para ello, la puesta en marcha de una correcta gestión del conocimiento es fundamental para optimizar tanto el uso de recursos y como la búsqueda de nuevas oportunidades de negocio para la empresa.

La intangibilidad del conocimiento hace que, al igual que sucede con la información, sea expandible, comprimible, sustituible, difusa y compartida (Aja, 2002). Un conocimiento y una información que han de ser procesadas con el auxilio de las TIC, formado por la combinación de Hardware y Software, de forma que se puede generar nuevo conocimiento a partir de la capacidad de innovación que tiene el ser humano (Wetware).

Dicha capacidad de aprender por parte de las organizaciones a partir del Wetware, junto a la generalización de las Nuevas Tecnologías de la Información y la Comunicación (NTIC), están llevando hacia un proceso de creación de alianzas estratégicas entre empresas (Saiz y Ollala, 2010). Este proceso se acelerará aún más en la Unión Europea con la formación de empresas transfronterizas tras la creación de las denominadas regiones europeas (NUTS, del francés Nomenclature des Unités Territoriales Statistiques) caracterizadas por la transmisión en tiempo real de información entre empresas situadas en distintos socios comunitarios, así como por el intercambio de capital intelectual entre las organizaciones. Para poder llevar a cabo este proceso se hace necesario que las barreras de entrada disminuyan y, en caso óptimo, se eliminen entre las organizaciones, en concreto las barreras técnicas, culturales (esto es, compatibilizar distintos usos, costumbres, horarios y formas de trabajo e interacción entre individuos, tanto en un sentido horizontal como vertical entre las organizaciones) e idiomáticas, con el establecimiento de una o varias lenguas de trabajo. En todo ello el conocimiento tiene una importancia fundamental. Conocimiento que se valoriza en términos de trabajo y productividad del capital humano que trabaja en la organización.

La experiencia europea en términos de colaboración empresarial, así como de la internacionalización de las grandes corporaciones una vez que éstas han alcanzado una masa crítica que les hacen capaces de sobrevivir en entornos hostiles, puede constituir un apoyo para unas empresas latinoamericanas que necesitan expandirse con eficacia y eficiencia en entornos internacionales. En este sentido, el capital intelectual que trabaja en dichas organizaciones tiene una importancia fundamental. De ahí que sea necesario implantar políticas de apoyo a dicho capital, tanto con sistemas de retribuciones basados en méritos y resultados, como en la puesta en marcha de políticas de conciliación laboral y carrera profesional. Políticas que, a su vez, han de llevar hacia una correcta gestión del conocimiento para que así la empresa, con independencia de su tamaño relativo y volumen de facturación, pueda crecer de forma sana en el tiempo y así alcanzar y, en su caso, mantener posiciones de liderazgo dentro del sector en el que compita, al ser el capital intelectual el activo más importante dentro de una empresa y el conocimiento un activo intangible ilimitado cuya importancia va creciendo con su uso. 


\section{REFERENCIAS BIBLIOGRÁFICAS}

Abdullah, R.; Sahibudin, S.; Alias, R.A. y Selamat, M.H. (2005).Collaborative Knowledge Management Systems for Learning Organisations., Journal of Information \& Knowledge Management, vol. 4, n. 4, p 237245.

Ackerman, M.; Pipek, V. y Wulf, V. (2003). Sharing Expertise: Beyond Knowledge Management, Boston: MIT Press Books.

Aja, L. (2002). Gestión de información, gestión del conocimiento y gestión de la calidad en las organizaciones. mimeo, Hospital Militar Central Dr. Carlos J. Finlay, Ciudad de la Habana (Cuba).

Alavi, M. y Leidner, D.E. (2001). Review: Knowledge Management and Knowledge Management Systems: Conceptual foundations and research issues. MIS Quarterly: Management Information Systems, vol. 25, n. 1, p 107-136.

Becerra-Fernández, I. y Sabherwal, R. (2010). Knowledge Management: Systems and Processes, Nueva York: Sharpe.

Ben-Porath, Y. (1967. The Production of Human Capital and the Life Cycle of Earnings, Journal of Political Economy, vol. 75, n. 4, p 352-365.

Bhatt, M. (2008). Managing Knowledge Management in Organizational Settings. Munich Personal RePEC Archive (MPRA), n. 30873, p 17-26.

Boisot, M. (1995). Information space: A framework for learning in organizations, institutions and culture, Londres (Reino Unido): Routledge.

Bradfield, D.J.; Gao, J.X. y Soltan, H. (2007). A metaknowledge approach to facilitate knowledge sharing in the global product development process", Computer-Aided Design and Applications, vol. 4, n. 1-6, p 519528.

Cristea, D. y Capatina, A. (2010). Knowledge Economy and the Necessity of Knowledge Management, The Annals of Dunarea de Jos, Universidad de Galati, Rumania, n. 1, p 181-188.

Davenport, T.H. y Prusak, L. (2000). Working Management: how organizations manage what they know, Harvard Business School.

Drucker, P.F. (1969). The Age of Discontinuity. Guidelines to our Changing Society, Transaction Publishers. 
Hall, R. y Andriani, P. (2003.Managing Knowledge associated with Innovation. Journal of Business Research, vol. 56, n. 2, p 145-152.

Heckman, J. J. (1976).A Life-Cycle Model of Earnings, Learning, and Consumption. Journal of Political Economy, vol. 84, n. 4, p S11-44.

Hiari, C.; Uchida, Y. y Fujinami, T. (2007). A Knowledge Management System for Dynamic Organizational Knowledge Circulation. International Journal of Information Technology and Decision Making, vol. 6, n. 3, p 509-522.

Hsu, I-C. y Sabherwal, R. (2012). Relationship between Intellectual Capital and Knowledge Management: An Empirical Investigation. Decision Sciences, vol. 43, n. 3, Junio, p 489-524.

(2011). From intellectual capital to firm performance: The mediating role of knowledge management capabilities. IEEE Transactions on Engineering Management, vol. 58, n. 4, Noviembre, p 626-642.

Hussain, I.; Ahmed, S. y Si, S. (2010). Personal Knowledge Abilities and Knowledge Management Success. Journal of Information \& Knowledge Management, n. 4, p 319-327.

Hussi, T. (2003). Reconfiguring Knowledge Management. Combining Intellectual Capital, Intangible Assets and Knowledge Creation", Discussion Papers, 849, Helsinki (Finlandia): The Research Institute of the Finnish Economy (ETLA).

Ion, A-M (2008). Knowledge Management and eLearning. Informática Económica, vol. 4, n. 48, p 80-100.

Jovanovic, B. y Rob, R. (1989). The Growth and Diffusion of Knowledge. Review of Economic Studies, vol. 56, n. 4, p 569-582.

Koenig, M.D.; Lorenz, J. y Zilibotti, F. (2012). Innovation vs. Imitation and the Evolution of Productivity Distributions. SIEPR Discussion Paper, n. 11-008, Stanford, Ca. (Estados Unidos): Stanford Institute for Economic Policy Research (SIEPR), Universidad de Stanford.

Kogut, B. y Zander, U. (1992). Knowledge of the firm, combinative capabilities, and the replication of technology. Organization Science, vol. 3, p 383-397.

Leung, Z.C.S.; Cheung, C.F.; Chan, K.T. y Lo, K.H.K. (2012). Effectiveness of Knowledge Management Systems in Social Services: Food Assistance Project as an Example. Administration in Social Work, vol. 36, n. 3, p 302-313.

Lucas, R.E. (2009). Ideas and Growth, Economica, vol. 76, n. 301, p 1-19.

McLuhan, M. (1989). The Global Village, Oxford University Press. 
Mishra, J.K. (2009). Knowledge Management: Complexity, Learning \& Sustainable, New Delhi (India): Innovation Global India Publications.

Mitja, T. (2011). The Knowledge Management Wheel. Knowledge as Business Opportunity. Proceedings of the Management, Knowledge and Learning International Conference 2011, 22-24 de junio, Eslovenia.

Mittal, M. (2008). Personal Knowledge management: A Study of Knowledge Behaviour of Academicians. Journal of Information \& Knowledge Management, vol. 7, n. 2, p 93-100.

Moran, P. y Ghoshal, S. (1996). Value creation by firms. En Keys, J.B. y Dosier, L.N. (Eds.), Academy of Management Best Paper Proceedings, p 41-45.

Nahapiet, J. y Ghoshal, S. (1998). Social Capital, Intellectual Capital and the Organizational Advantage. Academy of Management Review, vol. 23, n. 2, p 242-266.

Perraton, J. y Tarrant, I. (2007). What does tacit knowledge actually explains?, Journal of Economic Methodology, vol. 14, n. 3, p 353-370.

Polanyi, M. (1958). Personal Knowledge. Towards a Post-critical Philosophy, Londres (Reino Unido): Routledge.

Ricciardi, R.I.; Barroso, A.C.O. y Ermine, J.-L. (2005). Knowledge Evaluation for Knowledge Management Implementation-The Case Study of the Radio-pharmaceutical Center of IPEN', I International Conference on Nuclear Knowledge Management, INAC 2005, Santos (Brasil).

Romer, P. (1995). Beyond the Knowledge Worker, Worldlink, Enero/Febrero, p 23-32.

Rosen, S. (1976). A Theory of Life Earnings, Journal of Political Economy, vol. 84, n. 4, p S45-67.

Saiz, J.M. y Olalla, B. (2010). Gestión del conocimiento y sistemas de calidad en los clusters de empresas familiares. Revista Escuela de Administración de Negocios. No 68, enero-junio, Bogotá (Colombia): Universidad EAN. p 70-85.

Sene, I. y Berdugo, A. (2000): Éthique et Knowledge-Management, Cahier de Recherche, No. 707, École des Hautes Études Commerciales (HEC), Paris (Francia).

Urbano, D.; Díaz, J.C. y Hernández, R. (2007). Evolución y principios de la Teoría Económica Institucional: una propuesta de aplicación para el análisis de los factores condicionantes de la creación de empresas. Investigaciones europeas de dirección y economía de la empresa. Vol. 13, n. 3. Madrid: Academia Europea de Dirección y Economía de la Empresa, p 183-198.

Wang, Z. y Wang, N. (2012). Knowledge sharing, innovation and firm performance, Expert Systems with Applications, vol. 39, n. 10, p 8899-8908. 


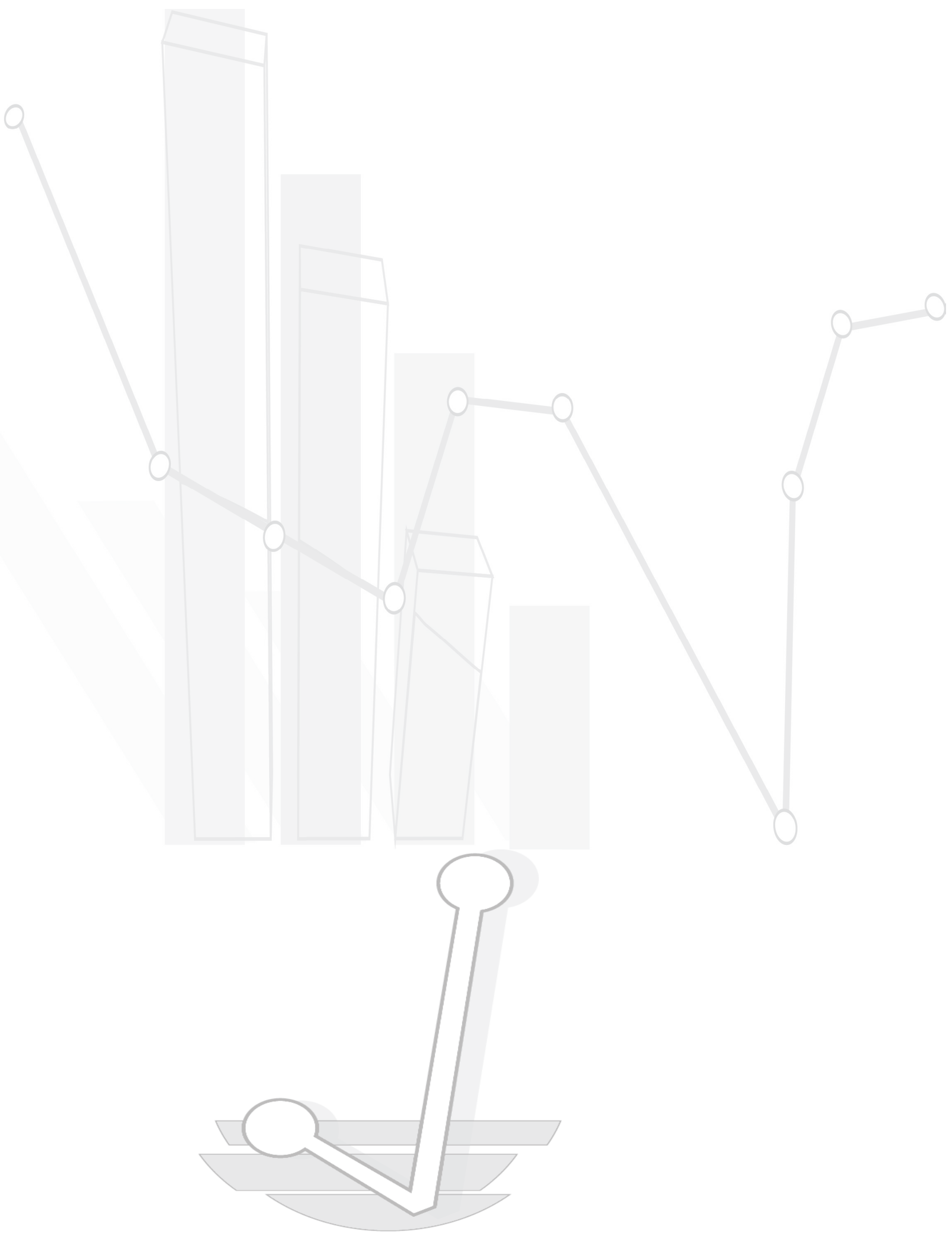

